\title{
Gallbladder Mucinous Cystic Neoplasm with Intermediate Grade Intraepithelial Neoplasia
}

National Cancer Institute

\section{Source}

National Cancer Institute. Gallbladder Mucinous Cystic Neoplasm with Intermediate

Grade Intraepithelial Neoplasia. NCI Thesaurus. Code C96884.

A non-invasive mucinous cystic neoplasm that arises from the gallbladder epithelium. It is characterized by the presence of moderate atypia. 